\title{
An Attempt to Define Fully-Accessible Workstation Levels of Accessibility
}

\author{
RJ Cooper \\ Adaptive Technology Specialist \\ Capistrano School District \\ San Juan Capistrano, CA, USA \\ Jeffrey C. Senge \\ Computer Access Coordinator \\ California State University at Fullerton \\ Fullerton, CA, USA \\ Contact information: \\ 24843 Del Prado \#283 \\ Dana Point, CA 92629 USA \\ Voice: 714-240-4853 Fax: 714-240-9785 \\ Email: rjcoop@AOL.com or jsenge@fullerton.edu
}

The development and adoption of defined and standardized 'fully accessible workstations', which serve the needs of a great percentage of persons that require non-traditional access to computers, is a goal of all facilities that consider meeting the needs of persons with disabilities. Although the needs of every person with a disability are unique to that individual, it is possible to develop standards based upon relative incidence and probable need of technology assistance. These standards would provide a known starting point for those facilities wishing to begin technology access assistance programs, and structured upgrade paths for those facilities wishing to expand their services to cover other disabilities.

\section{Introduction}

The authors suggest adopting a series of Fully-Accessible Workstation (FAW) standards, that would define those assistive technology aids necessary to provide computer access to various persons with non-traditional abilities. In the United States, at present, various groups are implementing technology access programs with a need to specify which type of product will address which type of disability, and relative need and necessity of each disability.

"Almost 1 in 11 full-time freshman (8.8\%) enrolled in college in 1991 reported a disability. Since 1987, this was a considerable increase when the proportion was about 1 in 38 freshmen (2.6\%)." [1] FAW accessibility must address prevalence and need, which are inversely proportional. Visual and learning disabilities comprise $25 \%$ of those freshmen listing a disability, while orthopedic disabilities comprise only 
14\% [1]. The need for assisted technology access is high for those with severe visual impairments, low and as yet unproven for those with learning disabilities, and high, once again, for those with severe orthopedic disabilities. Naturally, the greatest number of people with disabilities have moderate disabilities, which dictate less need, while the fewer number of people with disabilities have severe disabilities, which dictate greater need. That is, those with moderate disabilities have less of a need for technology access assistance while those with severe disabilities have a greater need. This applies to both computer course/work and to that course/work which can only be accomplished via computers by a person with a severe disability. As an example of the former, most English Composition course/work is executed via word processing; this can be considered computer coursework. An example of the latter may be researching a particular subject which requires physically locating, and manipulating numerous reference materials. This process simply cannot be performed manually by a person with severe disability. This person requires not only technology to perform the research, such as on-line reference databases, but technology assistance to access the technology which allows the research to proceed.

The precedent for such a system of Level definitions exists in the MPC (MultiMedia Personal Computer) Levels set by MicroSoft, IBM, Intel, and others. These Levels dictate what equipment is necessary for a computer company, or valueadded reseller, to label their product MPC Level One or Level Two. This pseudocertification addresses hard disk space, processor type and speed, sound capabilities, CD-ROM inclusion, and several other components. That is, one works along a continuum of possible devices employed in a home workstation, from very basic, to very high end, capable of handling any type of application.

This paradigm can easily be applied to workstations for persons with disabilities. After traditional keyboard access is considered inappropriate by either the end-user, care-giver, or facilitator (employee, technology specialist, or other person at the facility in question), one works along a continuum of devices and aids, from slightly assistive to greatly assistive. As the facility gathers more adaptive devices for the workstation, it becomes more capable of handling persons with a greater variety of disabilities.

These standards can be applied to any computer platform, and are not specific to brand. Adaptive devices for almost all computer types are now made commercially and available internationally. For the purposes of this paper, the authors will limit themselves to the two main platforms, the Macintosh by Apple Computer, and a PC, which is any computer that is IBM $80 \times 86$ or Pentium compatible.

In most cases, the authors are referencing a specific product by a specific company, but brand names and company names are omitted for academic reasons. The intent is to specify device capability, rather than product promotion. Should readers wish to receive references on specific devices, they should contact the authors.

\section{Methodology}

A continuum of physical abilities (for persons with DISabilities) and devices that address those abilities will now be presented. Beginning with those with the most moderate handicaps the list will ascend to those with the greatest challenges. This 
will take the form of specifying the physical abilities of the user, and the possible device and capabilities of the adaptive device.

For those persons that can access the keyboard, and can move a mouse, but cannot click the mouse while holding it steady:

1) A trackball which allows the user to move a ball within its cradle, remove his/her hand from the ball, and click with a different motion entirely is recommended.

2) An external switch, operated by another motion, other than the ball manipulation is recommended. This switch is hard-wired to emulate the mouse-click.

3) A second external switch is hard-wired to a button on the trackball which performs a 'drag-lock', that is, activating this second switch causes the computer to believe the user is holding down the primary click, thus performing the drag function.

4) This device shall be known as a Switch-Adapted Mouse device.

For persons that can access the regular keyboard but cannot control the mouse sufficiently:

1) A trackball might be appropriate.

2) A joystick that emulates the mouse might be appropriate.

a) This device shall be known as a Joy-Mouse.

For those persons that can access the keyboard, but cannot manipulate the mouse at all:

1) There are free programs available for both platforms that allow certain keys to move and click the mouse, so that all mouse functions can be performed by keyboard. Macintosh computers include these programs with their systems, while the corresponding utilities for PC's are available free from IBM.

2) These utilities shall be known as Mouse-Keys.

For those persons that have gross control of their hands but cannot access the traditional keyboard or mouse:

1) A slightly larger keyboard with 'keys' that have greater separation than the traditional keyboard, is recommended. This keyboard is also 'definable' so that it can be customized to a person's needs, physically, and software-wise. This keyboard is simply plugged in where the normal keyboard is usually plugged in, and the normal keyboard can still be plugged in tandem with it. This keyboard can also perform mouse functions by keystrokes.

2) This device shall be known as an Oversize Keyboard.

For those persons that have limited vision:

1) Several 'magnifying programs' exist which enlarge any portion of the screen selected by the user. The Macintosh ships with the program CloseView for this purpose. Similar programs are available for the PC.

2) These programs shall be known as Screen Magnification Software.

For those persons that can hear but cannot see the screen at all: 
1) Numerous software programs exist for the $P C$ and one is available for the Macintosh which give a blind person complete access to user-selectable auditory monitoring of both text-based and Graphic-User-Interface screens.

2) These programs shall be known as Screen Reading Software.

For those persons that can operate a pointing device but cannot access a normal keyboard or an oversize keyboard:

1) An On-Screen Keyboard program allows a person that can use a joystick, mouse, or a trackball, to move a cursor/arrow and click the button, in order to select keystrokes. Mouse moves can be handled directly through the appropriate input device or through appropriate keys on the on-screen keyboard.

a) These programs shall be known as On-Screen Keyboard Software.

2) There exists a variety of pointing devices. The best known is a mouse, of course, with a trackball and joystick also well known. On the Adaptive Technology market, there are several devices available for persons that can move another part of their body, most frequently their head, to control an on-screen cursor that tracks the movement of the controlling appendage.

3) Several of these on-screen keyboard programs allow the user to select keystrokes by simply by keeping the cursor over an on-screen key or function for a preset time.

a) This function shall be known as Dwell-Selectable.

For those persons that can operate a single switch, with a voluntary and consistent motor movement, but cannot access any of the above:

1) Some type of screen scanning is called for. With this method, the user clicks an appropriate external switch or input device to initiate an indicator which moves through the user's possible actions/selections, until the user activates their input device to send the desired selection to the host application. The following scenario illustrates this approach.

A horizontal line appears across the width of the screen, at the top of the screen. This line descends down the screen until it reaches the desired vertical location and the user clicks a second time. A finger appears at the left edge of the line and begins moving horizontally until it reaches the desired horizontal location. The user clicks a third time to select that location, just as if a traditional mouse mover moved the mouse directly to that location, and clicked. Double-clicks, drags, and menu bars are handled similarly. Text entry may be performed via an On-Screen keyboard by this same method, only restricting the area of screen scanning to the Keyboard area to increase speed of input.

\section{Corroboration}

The results of a survey concerning this matter will be disbursed at the conference. The body currently being polled are members of Project EASI, Equal Access to Software and Information, an international collaborative effort of professionals in higher education, representatives of several Fortune 500 companies, and developers of adaptive aids. One of the missions of Project EASI is to assess and evaluate the 
technology status of workstations as it currently relates to access and information. From this assessment and dialog, solutions are sought. Both authors of this paper are members of Project EASI. It is hoped that survey results of up to 100 members will be compiled and analyzed. These results, along with the authors' specifications, will define EASI/FAW Levels.

\section{Conclusions}

In deciding what part of its budget to allocate to assistive devices, a facility could compile data on prevalence and need relative to the populations it wishes to serve. After prioritizing the workstation needs as dictated by the compilation, the facility could simply cross-reference its needs with the FAW Level sequence to help isolate possible assistive aids. Further research would be necessary, by the facility, to decide between brands and features of products at a certain level, but it is hoped that many manufacturers would adopt the standards also, so as to make the facility's comparison efforts easier.

Each level specified herein would need to be further defined and possibly divided into sub-levels, as features become accepted as standards, or remain product features. That is, the primary features of a product apply to its Level assignment, whereas the secondary features of a product might be considered Preferences, which are available with most software. It will be quite difficult, without complete manufacturer agreement, to separate these two. A certain manufacturer might claim that their feature is really necessary to address a certain Level, whereas another manufacturer might claim that it is their feature that defines the standard at that level. Some sort of governing peer group would be necessary to make certification decisions, Level definitions, and guideline adherence.

Given the above paragraph's success, and acceptance of the theme of this paper, a committee within a group such as Project EASI could begin work on logistics. The following steps might form a desired sequence of activity.

1) Fully specify, along a continuum such as that presented here, all major assistive item groups and the disabilities they address.

2) Define those features of each item that form the basis of that Level.

3) Specify those items, of all items made, that conform to that Level specification.

4) Inform manufacturers of their inclusion or exclusion, and specify rationale.

5) Choose certain model sites and assist in implementation.

6) Publish articles in appropriate publications reporting creation of Level standards.

7) Produce FAW Level booklets specifying guidelines and criteria for facilities wishing to replicate model sites.

As an example of the difficulty facing such a project, the following quotations are presented from an E-mail letter attempting to negotiate/work out a compromise with the Computer Access Bill facing California legislators during midMay, 1994. The authors of this E-mail attempted to facilitate a compromise between needs and funds availability, and found that insufficient definitions existed to reach a compromise. [2]

"...this study shall include....an assessment of the degree to which students with disabilities are currently able to use the computers in each open lab." 
"An accessible computer is one that has been modified to contain hardware or software that makes it usable by a visually, mobility or hearing impaired student."

"This is accomplished primarily by consulting with individual students about the computer hardware and software accommodations they need, and providing access to adaptive computers..."

"We need to agree on a chart of what is needed for the computer station...and what an accessible station should look like. We do not need several chefs cooking this brew. We need consensus among us and especially among the high tech center program managers."

One can easily ascertain, from these quotations, the need for acceptance of this paper, or its theme. If one of the largest post-secondary school systems in the world, the California State University system, is having difficulties such as quoted above, then it is time to consider standardization such as those proposed herein.

\section{References}

1. C. Henderson: College Freshmen with Disabilities: A Statistical Profile. In HEATH Resource Center of the American Council on Education. Washington, DC.

2. California State Department of Rehabilitation: E-mail letter distributed to all University of California and California State University campus administrators 